The middle and ring fingers were strongly flexed, and the slightest attempt to extend them caused great pain. On examination a small bone or fragment of bone was discovered lying under the skin in the middle line of the front part of the forearm, about an inch above the flexure of the wi ist. It was thought to be a displaced comminution of the radius. It evidently interfered with the flexor tendons of the middle and ring fingers so much as to necessitate those fingers remaining in a clawed position. It also pressed on the median nerve, causing a numbness of those parts of the skin to which that nerve is supplied. I, therefore, cut down upon it with antiseptic precautions, and it proved to be the entire semilunar bone dislocated into this strange position. After its removal the wound was treated antiseptically, and healed without any suppuration, the patient being dismissed with a good useful hand and wrist.

CASE 7. Fracture of the Base of the Slcull, in which Cerebro-spinal Fluid escaped freely from the left Nostril and Ear. - In cases of fracture of the base of the skull, clear fluid is frequently observed, at some period of their progress, to escape in greater or less quantity from one or both ears; this fluid being now understood to be, at least in the majority of cases, cerebro-spinal fluid. The same liquid has been noticed very occasionally to exude from compound fractures of the vault of the cranium; and in apparently still fewer cases, to drain away from one or both nostrils. This was first observed by Blandin in the Hotel Dieu in 1840. In 1845 Robert met with a case where there was a great discharge from the nostrils during life and also after death, on the body being placed on its face. Examination revealed fracture of the sella turcica with laceration of the dura mater and the visceral arachnoid over the anterior lobes of the brain. Cases are also recorded by Goucard, Malgaigne, Erichsen, and perhaps others. The details of my case are as follows :- - H, P-, aged seventy-five, was brought to the Royal Infirmary on May 8th, 1876, having been assaulted and violently thrown down a stair. He was not quite unconscious, but incoherent and excited in talk, and unable to walk, staggering and falling if set upon his feet. There was profuse bleeding from his left ear and nostril, followed by the discharge from both of large quantities of clear fluid. In the course of the day the left eye also became closed by subconjunctival extravasation of blood, both palpebral and ocular. The discharge of cerebro-spinal fluid continued for several days, ceasing from the ear before it did so from the nostril. His recovery was tedious, but at last, in spite of his advanced age, was complete and satisfactory. I think there is little doubt that in this case there existed a fracture ex tending through the petrous portion of the temporal bone, laceratiog the process of arachnoid which accompanies the auditory nerve in the internal auditory canal, passing through the orbital plate of the frontal bone and also through the cribriform plate of the ethmoid, and opening the prolongation of the arachnoid that surrounds the filaments of the olfactory nerve, thus permitting escape of subarachnoid fluid both by the ear and nostril and of blood into the cellular tissue of the orbit. (To be concluded.)

\section{NOTES ON THE}

\section{DIAGNOSIS OF HYDATID CYSTS. BY JONAS JONASSEN, M.D.}

THE late Dr. Hjaltalin, as is well known, was the author of several articles on various diseases of Iceland, amongst which I may particularly refer to those on the treatment of hydatid disease. With all due respect for my late friend and chief, I find that his statements with regard to the prevalence of this malady in Iceland are at variance with facts, and that medical men such as the late Dr. Leared have been so far misled as to give currency to the exaggerated assertion that every fifth individual in Iceland succumbs to this affection.

As many of my professional brethren abroad are aware, this dreadful malady is peculiar to Iceland; therefore I thought it might be of interest to medical men in England to know what I had to say on the subject. My observations are founded on fifteen years' practical experience, and in $\mathrm{my}$ opinion many of Dr. Hjaltalin's assertions will fail to stand the test of the analysis of that experience. If this contribu- tion, based as it is on long practice, should find favour in England, I intend to follow it up with further observations, not only as to the peculiar nature of this disease, but also as to the practical treatment which I have adhered to and found more successful than any other. It is a natural consequence of the sporadic appearance of this disease in other countries (Australia excepied) that medical men have not had under observation sufficient material from which to acquire the necessary knowledge of the malady, neither at the bedside nor by autopsy, and therefore we search in vain for any strict analysis of the many diagnostic difficulties which the disease itself offers in so many of its various stages. I intend to offer some suggestions in illustration of this par. ticular subject.

The diagnosis of the existence of a hydatid cyst is sometimes very easy, but at others highly difficult, if not impossible. It is, as it were, merely dependent upon the physical examination; for, apart from those symptoms which are obtained by the physical examination, there does not exist a single symptom particularly characteristic of the hydatid disease. Of the various autopsies which I have undertaken myself, I have in many cases found cysts of the existence of which I had no idea. In consequence of the frequent occurrence of hydatid disease in Iceland, and the rarity of other diseases of the liver, Icelandic practitioners are, in reality, less liable to mistakes as to the diagnosis than they would otherwise be, Although in Iceland the medical man seldom fails to recogaise a palpable hydatid cyst, it is, on the other hand, none the less a fact that the older doctors in many cases have based their diagnosis on single symptoms before any tumour was observed by the objective examination; hence they have founded their diagnosis on uncertain premisses, and this is the cause of their frequent exaggeratiors as to the extensive prevalence of the disease in Iceland. ${ }^{1}$ What I wish emphatically to insist on is, that the existence of the disease itself can be proved pretty accurately, when the tumour presents itself, by examination. The hydatid cysts develop gradually, so that a long time may elapse before the appear ance of the tumour, and as the liver cysts are most frequent, and these usually develop in the posterior part of the liver, the least accessible portion for examination, one may be left for a long time in uncertainty as to the real existence of the dizease.

The diagnosis between liver cyst and cyst in the abdomen outside of the liver is, in many cases, extremely difficult, often even impossible. It sometimes happens that one finds a tumour in the hepatic region, which may favour the supposition that the cyst is in the liver itself. On post-mortem examina. tion, however, it is discovered that the cyst is quite in dependent of the liver; in fact, issues from, and is connected with, the abdominal wall. As a rule, when the cyst has its seat in the upper part of the abdomen, it is impossible to be certain whether it is in, or outside of, the liver. Still more uncertain is the diagnosis when the cyst has become so large as to fill the greater part of the abdo. minal cavity. If, however, the cyst has its seat in the middle part of the abdomen, or if it has not increased to any great size, it is possible to diagnose with approximate certainty an abdominal cyst outside of the liver. I would however, call attention to the fact that liver cysts are sometimes attached by merely a pedicle to the liver, and thus may reach far down in the abdominal cavity. Now I shall specially refer to cysts found in the lower part of the abdomen, and where it is impossible to say whether they go down in the pelvic cavity or not.

* As to the liver cyst specially, the diagnosis of whether it is situated in the right or left lobe of the liver cannot be ascertained with certainty during the patient's life; but my autopsies have proved that the right lobe is a locus electus, whilst the left lobe is the seat only in exceptional cases.

I shall now point out some other difficulties in diagnosis, which it is of great importance to overeome before any operative treatment is instituted-difficulties which scarcely any of the authors on this subject have yet brought under norice, - I mean those difficulties which present themselves when liver cyst has to be dealt with, and the aim of the practitioner is to arrive at a correct knowledge of the relationship of the cyst to the parenchyma of the liver-or, in other words, how large a part of the parenchyma has been destroyed by the cyst, and where the

1 As one instance among many may be mentioned Professor Wilson Edinburgh Health Irectures, 2nd series, 1882, pp. 69-70. 
boundaries between it and the remainder of the liver parenchyma are to be found. The cyst will excavate or destroy gradually the liver under its slow development, and hence a larger or smaller portion of the cyst is embedded in the mass of the liver. When the cyst develops itself from the posterior surface of the liver, it is not accessible for examination before it has reached the abdominal wall. As the cyst grows, the liver is depressed, and the hepatic dulness is removed, and one frequently finds the lower margin of the liver at a greater or less distance below the margin of the ribs, more or less obliquely placed in the abdomen. During this growth of the cyst more or less bulging of the lower part of the right chest is observable, also often a prominence over the region of the heart.

If the cyst has its seat in the right lobe of the liver, its development may take place downwards, outwards, and forwards, or downwards, inwards, and forwards, towards the linea media. By-and-by the liver is depressed further and further in the abdomen, and the cyst will in the first case come in contact with the abdominal wall, in or below the right hypochondrium. Thus it will be the cyst itself which forms the highest part of the tumour below the margin of the ribs; and this tumour usually shows the objective signs, which I shall refer to later on, while the lowest part of the tumour, which, as a rule, is more resistant, forms the remainder of the liver, provided it is not altogether destroyed by the cyst. In the other case, where the development has taken place more downwards, inwards, and forwards, the cyst will gradually come in contact with the abdominal wall in the upper part of the epigastric region, thrusting the left lobe, sometimes largely hypertrophied, upwards to the left. When the development of the cyst has taken place as described, which can only be known when one has had the opportunity of examining the patient repeatedly during his illness, then an operation may be attempted without risk of touching the liver, care being taken to operate only on the most prominent point of the tumour. I would, however, remark that sometimes it is quite impossible to determine with certainty whether it is the liver itself or the cyst which forms the most prominent part of the tumour; the capsule of the cyst may be very thick and compact, which makes the cyst feel like a solid tumour; and in addition to this a part of the liver may form a layer outside the cyst. In diagnosing the extent of the cyst, care must be taken not to be misled by the sharp and round margin, which often is to be felt through the abdominal wall, as this margin is sometimes the lowest boundary of the cyst itself.

If the cyst is most prominent in the upper part of the epigastrium, one is usually sure to come upon the cyst itself, not the parenchyma of the liver; however, it is possible to meet with cases where the prominence is not particularly developed, and in its stead is found a bulging, the boundary of which it is difficult to ascertain, and where the diagnosis between the cyst and the parenchyma of the liver is very difficult. When the cyst develops from the anterior part of the right lobe of the liver, the physical examination will give the same result as when it develops from the posterior part. Hence at this stage of the development, especially if the patient be examined for the first time, it is impossible to diagnose whether the cyst has originated from the posterior or anterior surface of the liver; this, however, is as a rule of slight importance. Of such a nature as I have now described I have found most cases of liver echinococci in Iceland. Only in rare instances has the development taken place from the left lobe of the liver, and in those cases one will find the cyst mostly prominent in the uppermost part of the epigastric region or the left hypochondrium. Further, I may call attention to those rare cases where the cyst develops from the posterior part of the liver, but takes an apward direction, in which the diaphragm is pressed more and more upwards, even towards the second intercostal space, at the same time the cyst, under its progressive growth, presses upon its surrounding parts. In such cases one of two things will happen : either the right side of the chest bulges out, without the liver being to any extent depressed; or else the liver is depressed in the abdomen, while the right side of the chest almost maintains its natural form. In the first instance the percussion note of nearly all the right side of the chest is quite dull, and this dulness extends a little below the normal boundary of the liver; in the latter instance the percussion note is the same, but the dulness extends further down in the abdomen. In the former case it is very doubtful whether one has to deal with a hydatid cyst at all, or, supposing such to be the case, whether the cyst is situated above or below the diaphragm. Very seldom the cyst is found to bave its seat on the lower level of the liver; in such cases the cyst may press upon the portal vein, the vena cava, or the larger bile-ducts, and as a consequence produce ascites with or without œdema of the lower extremities or icterus. When, however, there is reason to suppose that these symptoms arise from a hydatid cyst, it is natural to believe that the cyst, inaccessible to examination, has its seat in the lower surface of the liver. In cases of ascites, with or with. out cedema of the lower extremities, it is necessary first to make a puncture for ascites. I have myself observed two cases where the liver, after the puncture, presented itself as the seat of cysts, and this was clearly observable not only by palpation, but even to mere inspection; that in these cases there must have been a cyst, inaccessible to objective examination, on the concave part of the liver, which pressed on the vasa, there was every reason to assume, and in one of my cases my diagnosis was fully confirmed at the autopsy. In rare instances more than one cyst may be diagnosed in the liver, in which cases there may be several small ones of about the same size, producing an uneven and knotty appearance of the liver, or they may be fewer and larger. If the bulging of the hypochondrium continues for some time after a diagnosed cyst in the liver has been emptied, the cause may be either the presence of more un. diagnosed cysts, or the persistence of the disturbance resulting from the surroundings of the emptied cysts (for instance, in the case of a great hypertrophy of the remaining part of the liver); lastly, the cause may be that the capsule of the emptied cyst is extraordinarily thick and resistant. It is when the bulging of the hepatic region has continued for a long time, other dubious symptoms being added thereto, that one can with approximate certainty assume that this is caused by the presence of more cysts inaccessible for examination. Before operating it is impossible by diagnosis to decide as to the nature of the contents of a hydatid cyst, whether they be clear or purulent, because one cannot by the severity of the pain, or by the appearance of the patient, or by general feeling guess the inflammation of the sac; $m y$ experience proves, too, that one can neither by the presence nor by the absence of fremissement conclude whether the cyst contains daughter cysts or not; and the statement of Frerichs, that from the quantity of the discharge it is possible to tell whether the cyst contains daughter cysts or not, is incorrect, as I could prove by many instances which have come under my notice did space permit.

It is of great importance to ascertain whether the cyst adheres to the abdominal wall before any operation is undertaken. Budd advises that the boundaries of the tumour and the lower margin of the liver be traced out while the patient is lying on the left side, and then, by changing the position, and by deep respiration, to ascertain whether the boundaries change or not. Bonnet points out that the most prominent point of the cyst does not change its position by the alteration of the position of the patient, but is always exactly equally fluctuating. According to my experience neither of these plans is trustworthy. In the first place, it is highly difficult, and in many instances impossible, to map out clear boundaries for the cyst or determine its connexion with the liver; secondly, as regards the mobility of the cyst, it varies greatly, according as the seat is a parenchymatous organ, as the liver, or the abdominal cavity outside the liver. While the liver cyst is of small extent, there can never be any question about mobility, except in connexion with the liver itself; if the cyst be superficial, and if it have become rather extensive, then the mobility can sometimes be recognised, principally in those rare cases where the cyst issues from the liver with a pedicle ; if, however, a large liver cyst has to be dealt with, the mobility is, as a rule, limited, and the manifestation of it vanishes in most cases entirely by the expansion of the abdominal wall. This is also the case with great abdominal cysts outside the liver. On the other hand, the superficial abdominal cysts of smaller extent are mostly movable, but as they gradually increase their mobility diminishes. The question, therefore, arises, Is it possible, from the mobility or non-mobility of a cyst, with certainty to state whether it be adhesive to the abominal wall or not? The answer is, Great mobility of the cyst excludes with certainty the possibility of adhesion to the abdominal wall ; loose ad hesion, on the other hand, is compatible with slight mobility of cyst, 
as $I$ have ascertained by autopsy. Immobility of the cyst is, however, not at all a certain proof of adhesion to the abdominal wall. As to the liver cysto, their adhesion to abdominal walls, as before mentioned, cannot at all be recognised except in connexion with the motion of the liver itself; with regard to other liver cysts, the immobility may partly depend upon the before-mentioned expansion and partly upon the adhesion of the cyst to surrounding organs other than the abdominal wall. This is also the case in respect to the abdominal cyst inside the liver. I have had one case where, through the immobility of the cyst, I diagnosed it to be an abdominal eyst outside the liver, strongly adhesive to the abdominal wall. This cyst did not come under treatment, but the antopsy proved that it had no connexion whatever with the abdominal wall, but was strongly adhesive to the intestines.

In intimate connexion with the mobility of the cyst is the mobility of the abdominal wall over the cyst. Here the diagnosis is very difficult when the abdominal wall is thick; if, on the contrary, it happens to be thin, one can, by its mobility, with tolerable certainty determine whether the cyst is adhesive or not. According to my experience, there is no unmistakable sign of adhesion of the cyst to the abdominal wall, and even in those cases where such adhesion appears highly probable, it is impossible to determine whether all that part of the cyst, or only a small part of it which is adjoining to the abdominal wall, is adberent or not; or, in the latter instance, where the adhesion really does exist, which of course would be of the greatest moment in view of treatment. To this conclusion I have been led by the results of the autopsies I have had the opportunity of performing.

\section{ACUTE INFLAMMATION OF THE OCCIPITO ATLANTAL JOINTS ACCOMPANIED} BY PY AEMIA.

DEATH ON THE FIFTEENTH DAY.

By CHARLES A. BALLANCE, M.S. LoND.

H. $\mathrm{K}-$ - aged six years, came into my out-patient room on June 27 th, 1883 . His attitude at once attracted atten. tion as being pathognomonic of disease of the upper cervical spine. The boy stood erect with the arms pressed against the body and the hands in the trousers pockets. The spine was quite rigid, the head and neck moving only with the rest of the body. On touching the head the child cried out, and slight pressure on the vertex or any attempt at rotation caused great pain. On examining the back of the neck some fulness and marked tenderness were found to exist on either side of the median line in the region of the occipitoatloid joints. On laying the hand upon this part it was evident that the temperature was raised above that of the surrounding skin. Pressure upon the cervical spines was not very painful. When placed in the supine position the little patient could not rise without first turning over on his knees and then supporting his bead with both hands. Nothing abnormal was detected in the pharynx. The boy was fair, his countenance pale and anxions, the skin hot, and the temperature in the axilla $1005^{\circ}$. He was illnourished and anæmic. The history obtained from the mother was very brief. She said that four days before, on coming back from school the boy complained of pain in the neck and kept his head quite stiff, and that the pain and stiffness had increased and had prevented sleep at night. No history of any injury could be elicited. He had never been ill before. There was no family history of tubercle, rheumatism, or joint disease. The child was taken at once into the hospital, and his head was fixed by sandbags and pillows, which appeared to give him great comfort. The temperature in the evening rose to $1025^{\circ}$. The subsequent clinical record shows a very rapid down ward progress. It is shortly as follows: The next day, June 28tb, the temperature varied from $102 \cdot 5^{\circ}$ to $1035^{\circ}$. The boy had had no sleep, and was constantly crying. The position of most ease was obtained when the head was dependent the boy preferring either to sit with his head downwards between his knees, or to lie in the prone position with his head over the side of the bed, supported by his hands. Quinine and opium were ordered, and the spine and head fixed and supported by a splint, which, bowever, had to be removed two days afterwards in consequence of the extreme tenderness of the skin. -.July Ist: The swelling which had been noticed on either side of the upper part of the neck had become much more pronounced. - 3rd: The whole neck was edematous and greatly swollen, and the points from which the iuflammatory process had spread were very hard. Into this brawny region (on either side of the neck) an incision was made and carried down until the knife touched the transverse processes of the atlas. The wounds were then probed as deeply as possible. No pus was evacuated, but in a few hours a great deal of serum had oozed out, and in this way relief was affurded. -4th: The neck had almost resumed its normal size, but the patient was no better. The temperature remained above $103^{\circ}$, and the pulse was rapid and weak.5th : The left elbow-joint was painful, and there was evident effusion. -6th: The soft parts in the neighbourhood of the left elbow-joint pitted on pressure, and a distinct red blush was seen over the front and inner side of the joint. The tongue was dry, the patient drowsy, and some difficulty was experienced in obtainiog answers to questions. -7 th: The boy was very much worse. The inflammatory blush over, and the effusion in, the left elbow.joint had increased, There was an excoriated spot over each internal malleolus, aod over the back of each heel. Below each nipple was a fluctuating subcutaneous swelling. 'There were three similar tumours at the back of the trunk, one over the spine of the riuht scapula, and two over the spines of the vertebræ in the lumbo-sacral region. There were also fluctuating swellings in the right axilla, and under the scalp in the occipital region. The latter was incised, and a considerable quantity of pus evacuated, in order to give relief from the pain due to the weight of the head upon it. The temperature was $103.5^{\circ}$, the pulse 130, the skin yellowish, the bowels relaxed, and the tongue dry and cracked.-8th : The little patient became unconscious in the morning, and died at 5 P.M., fifteen days from the first symtoms of illness. The temperature had remained throughout continuously high, with only slight morning remissions, and during the pyæmic state neither rigors nor convulsions occurred.

The post-mortem examination was made the next day. The occipito-atlantal joints were carefully exposed by dissection. Their capsules were found to be distended, and when an incision was made into them thin fetid pus escaped. The cervical spine was then removed with the occipital bone for further examination; which revealed the fact that these joints were completely disorganised. The synovial membrane was swollen and injected. The cartilage had for the most part disappeared, the small portions remaining being closely adherent to the bone beneath. The bony surfaces entering into the joints which -had become exposed by the solution of the cartilage were superficially carious. The occipito-atlantal joints were alone unvolved in the disease. That no old disease of bone existed in their neighbourhood was proved by the inspection of sections made through the diseased joint surfaces. The other chief points elicited in the post-mortem examination were as follows :--The medulla oblongata and its membranes were normal. Pus was found in the left elbow-joint, and in the subcutaneous swellings over the front and back of the trunk, and in the right axilla. There was an abscess cavity over the occiput under the scalp which had not laid bare the bone, and which had no communication with the capsules of the occipito-atloid joints. No other pyæmic abscesses were discovered except in the lungs.

Remarks. - I have no doubt that the case related above is a rare one. The chief interest centres in the question, From what point did the pyæmic process start? Was the disease in the occipito-atlantal articulations the cause of or only one of the manifestations of the septic state? There are three ways in which the pyæmia may have been lighted up-1. By some source of infection, undiscovered at the post-mortem examination. This fallacy cannot wholly be eliminated, as the primary source of a pyæmia may remain latent during life and undiscovered after death, in consequence, probably, of the attention being diverted by the severity of the secon. dary inflammations. 2. By chronic mischief, periosteal or bony, in the neighbourhood of the affected joints. No such disease was, however, found during a careful examination of the cervical spine. 3 . By the acute mischief proceeding in the occipito-atlantal joints. The arguments which uphold this description of the etiology of the pyæmia are these$(x)$ Very distinct evidence of acute disease in these articula tions existed for twelve days before constitutional infection occurred. I was sure from my first examination of the boy 